\section{CARACTERIZACIÓN GENÉTICA DEL VIRUS DE VARICELA ZOSTER AISLADOS DE CASOS CLÍNICOS COMPLICADOS EN PERÚ EN 2016 Y 2017}

\author{
GENETIC CHARACTERIZATION OF \\ VARICELLA ZOSTER VIRUS ISOLATED FROM \\ COMPLICATED CLINICAL CASES IN PERU IN \\ 2016 AND 2017
}

Fredy J. Condori-Yujra ${ }^{1, a}$, Víctor A. Jimenez-Vasquez ${ }^{2, b}$, Giancarlo W. Enríquez-Alva ${ }^{3, c}$, Néstor E. Cabezudo-Pillpe $e^{1, d}$

Sr. Editor. En los últimos años en el Perú se presentó un alarmante aumento de casos por varicela complicada en población pediátrica. El laboratorio de referencia nacional de Sarampión y Rubéola del Instituto Nacional de Salud (INS), como parte de sus funciones de diagnóstico especializado, conservó muestras clínicas provenientes de pacientes diagnosticados con varicela. Las muestras correspondieron a suero sanguíneo e hisopado de lesión vesicular obtenidas de casos clínicos de un brote epidémico ocurrido en las regiones Lima, La Libertad, Loreto, Cusco y Tumbes. Las muestras fueron conservadas en refrigeración entre dos y ocho ${ }^{\circ} \mathrm{C}$, hasta su posterior procesamiento por ensayo por inmunoabsorción ligado a enzimas (ELISA) para determinar la respuesta de anticuerpos IgM, IgG; y por reacción en cadena de la polimerasa (PCR) en tiempo real para la detección del virus. Las muestras positivas fueron sujetas a un posterior análisis de caracterización molecular con el objetivo de genotipificar los virus de varicela zoster (VZV).

Entre el 2016 y 2017 fueron remitidas al laboratorio de Sarampión y Rubéola un total de 198 muestras de suero y 177 de hisopado de lesión vesicular. Del total de muestras 126 resultaron IgM positivos, y 104 fueron positivas en el PCR en tiempo real, las cuales fueron inoculadas en cultivos puros de células de Vero y Neuroblastoma. Las células

\footnotetext{
Laboratorio Nacional de Sarampión y Rubeola, Centro de Nacional de Salud Pública, Instituto Nacional de Salud. Lima, Perú.

2 Facultad de Ciencias Biológicas, Escuela Profesional de Ciencias Biológicas, Unidad de Sistemática Molecular y Filogeografía. Universidad Nacional Mayor de San Marcos. Lima, Perú.

3 Facultad de Ciencias Naturales y Matemáticas, Escuela Profesional de Biología, Universidad Nacional Federico Villareal. Lima, Perú.

a Biólogo Microbiólogo, especialista en Genética y Biología Molecular; ${ }^{\mathrm{b}}$ Biólogo, especialista en Biología Molecular; ${ }^{\mathrm{C}}$ Biólogo; ${ }^{\mathrm{d}}$ Biólogo Microbiólogo, especialista en Epidemiología.

Recibido: 28/05/2018 Aprobado: 15/08/2018 En línea: 23/08/2018
}

Citar como: Condori-Yujra FJ, Jimenez-Vasquez VA, Enríquez-Alva GW Cabezudo-Pillpe NE. Caracterización genética del virus de varicela zoster aislado de casos clínicos complicados en Perú en 2016 y 2017. Rev Peru Med Exp Salud Publica. 2018;35(3): 533-4. doi:10.17843/rpmesp.2018.353.3706. experimentaron un efecto citopático como consecuencia de la infección producida por VZV. Posteriormente los aislamientos fueron cosechados y almacenados a $-20^{\circ} \mathrm{C}$; a partir de estos se realizaron las extracciones de ADN usando el kit comercial QIAamp DNA Mini (QIAGEN), seguidamente se realizaron las amplificaciones por $\mathrm{PCR}$ convencional con el uso de cebadores (primers) desarrollados por Li \& Zhu para VZV y para las siguientes regiones: ORF1, ORF 21, ORF 22, ORF 38, ORF 54, ORF 62 y R5 ${ }^{(1)}$.

Los resultados fueron observados en geles de agarosa al $2 \%$, los productos de PCR se purificaron usando un kit comercial de $A D N$ genómico QIAquick Gel Extraction (QIAGEN) y fueron secuenciados por el método de Sanger y procesados en un analizador genético Applied Biosystems 3500. Se obtuvieron cromatogramas de ambas hebras (forward y reverse) los cuales fueron editados y ensamblados con el programa SeqTrace versión 0.9.0 ${ }^{(2)}$, las secuencias obtenidas fueron luego contrastadas con secuencias de genomas completos o parciales depositadas en la base de datos GenBanK ${ }^{(3)}$, de la que se descargaron 165 para cada región estudiada (ORFs). Se realizaron alineamientos de secuencias con el programa MAFFT versión $7^{(4)}$ y la concatenación de todas las regiones con el programa Sequence Matrix $1.8^{(5)}$. El alineamiento total estuvo conformado por una matriz de 171 secuencias y 3456 pares de bases (pb). Se identificaron los cambios a nivel nucleotídico y aminoacídico de las muestras peruanas con respecto de la vacuna VariVax. Se realizó una reconstrucción filogenética con la metodología NeighborJoining $(\mathrm{NJ})$ con la opción pairwise deleción, modelo de sustitución Kimura 2 parámetros (K2P), y 1000 réplicas bootstrap. Estas dos últimas operaciones fueron realizadas con el programa MEGA $7^{\left({ }^{(6)}\right.}$.

El análisis de las secuencias nucleotídicas obtenidas a partir de las extracciones de ADN de los cultivos celulares, permitió determinar la presencia del VZV en las muestras, confirmándose los resultados previos de PCR en tiempo real. Como parte de la genotipificación de VZV se obtuvo un árbol filogenético $\mathrm{NJ}$ en el que se distinguen los clados a los que pertenecen las muestras analizadas (Figura 1). Se registraron 29 cambios nucleotídicos puntuales (SNPs) que se traducen en 13 cambios no sinónimos, es decir, cambios aminoácidicos con respecto de la vacuna circulante VariVax, resaltada como triangulo negro en el clado 2 de la Figura 1.

Resaltamos que la topología del árbol NJ se asemeja (aunque no es igual) a la obtenida por otros autores con datos de genomas completos, dado que se distinguen los clados $1,2,3,4,5, \mathrm{VI}, \mathrm{VIII}$ y IX como grupos independientes, asimismo, los soportes bootstrap de cada clado superan el $50 \%$ considerado como límite ${ }^{(7,8)}$. La mayoría de las cepas colectadas entre 2016 y 2017 se agrupan en el clado 


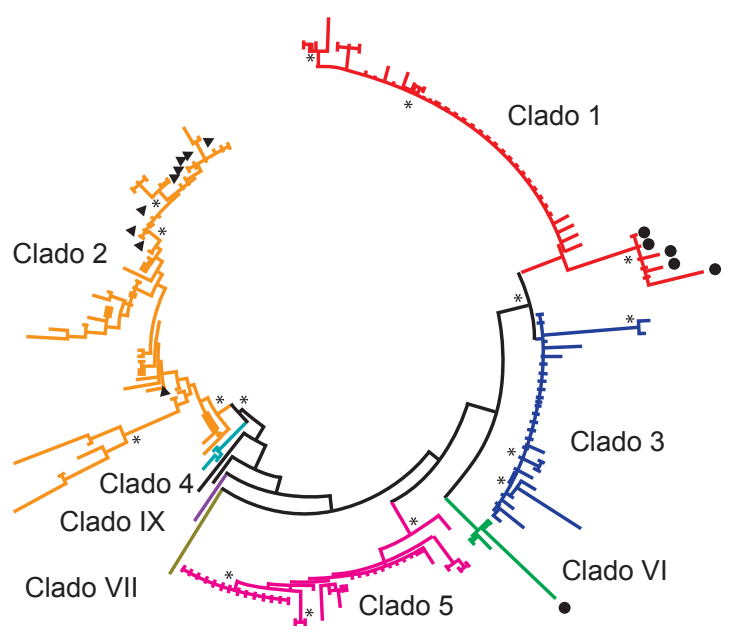

Figura 1. Árbol Neighbor-Joining de las relaciones evolutivas de Virus de Varicela Zoster obtenido de la concatenación de las regiones génicas ORF 1, ORF 21, ORF 22, ORF 34, ORF 54 , ORF 62 y R5. Se resaltan todos los clados identificados, los círculos corresponden a las muestras peruanas. Los triángulos corresponden a las vacunas pOka, vOka, VariVax, los asteriscos sobre cada nodo indican valores bootstrap mayores a 50 .

1 (en rojo en la Figura 1), el mismo que está conformado por cepas circulantes en Alemania, Suecia, Reino Unido, EE.UU. y forman un sub-clado independiente; en tanto, solo una cepa pertenece al clado 6 (en verde en la Figura 1).

Las muestras peruanas aisladas entre 2016y 2017 pertenecen a dos grupos genéticamente diferenciados (clados 1 y VI). Determinamos que las muestras peruanas con respecto a la vacuna VariVax presentan 29 cambios nucleotídicos y 13 a nivel aminoacídico. Las siete regiones empleadas para el análisis filogenético permiten caracterizar molecularmente las muestras peruanas almacenadas en el laboratorio de Sarampión y Rubeola; y podemos afirmar que corresponden a VZV. Sin embargo, mediante la secuenciación de alto rendimiento de todo el genoma, en el contexto de grandes virus de $A D N$, los datos recientes sugieren que los VZV también podrían considerarse candidatos para el modelado de la dinámica evolutiva de las poblaciones de estas especies y la diversidad dentro del huésped.

Agradecimientos: A Sara Martha Gordillo Vilchez e Irma Atalaya Atalaya.

Descargo de responsabilidad: las opiniones y afirmaciones contenidas aquí son propias de los autores y no deben interpretarse como posición oficial del Ministerio de Salud del Perú. Algunos de los autores de este trabajo (FJCY, NECP) laboran en el Centro Nacional de Salud Pública (CNSP) del Instituto Nacional de Salud (INS). El presente trabajo ha sido preparado como parte de sus funciones oficiales.

Fuente de financiamiento: el estudio fue financiado por la vigilancia de Sarampión y Rubéola (Instituto Nacional de Salud).

Conflictos de interés: los autores declaran no tener ningún conflicto de intereses.

\section{REFERENCIAS BIBLIOGRÁFICAS}

1. Li Y, Zhu B. Genotyping of clinical varicella-zoster virus isolates collected from Yunnan in Southwestern China. Biomed Rep. 2016; 4(2):209-14. DOI: 10.3892/br.2015.562.

2. Stucky BJ. SeqTrace: A Graphical Tool for Rapidly Processing DNA Sequencing Chromatograms. J Biomol Tech JBT. 2012; 23(3):90-93. DOI: 10.7171/jbt.12-2303-004.

3. Benson DA, Cavanaugh M, Clark K, Karsch-Mizrachi I, Ostell J, Pruitt KD, et al. GenBank. Nucleic Acids Res. 2018; 46(D1):

4. Katoh K, Misawa K, Kuma K, Miyata T. MAFFT: a novel method for rapid multiple sequence alignment based on fast Fourier transform. Nucleic Acids Res. 2002; 30(14):3059-66.

5. Vaidya G, Lohman DJ, Meier R. Sequence Matrix: concatenation software for the fast assembly of multi-gene datasets with character set and codon information. Cladistics. 2011; 27(2):171-80.

6. Kumar S, Stecher G, Tamura K. MEGA7: Molecular Evolutionary Genetics Analysis Version 7.0 for Bigger Datasets. Mol Biol Evol. 2016; 33(7):1870-4.

7. Norberg P, Depledge DP, Kundu S, Atkinson C, Brown J, Haque $\mathrm{T}$, et al. Recombination of Globally Circulating Varicella-Zoster Virus. J Virol. 2015; 89(14):7133-46.

8. Zell R, Taudien S, Pfaff F, Wutzler P, Platzer M, Sauerbrei A. Sequencing of 21 Varicella-Zoster Virus Genomes Reveals Two Novel Genotypes and Evidence of Recombination. J Virol. 2012; 86(3):1608-22.

Correspondencia: Fredy James Condori Yujra.

Dirección: Calle Cápac Yupanqui 1400, Jesús María, Lima, Perú. Teléfono: (511) 7481111.

Correo electrónico:fcondori@ins.gob.pe

\section{Lutzomyia shannoni UN POTENCIAL VECTOR DE Leishmania chagasi EN MADRE DE DIOS, PERÚ}

\section{Lutzomyia Shannoni A POTENTIAL VECTOR OF Leishmania chagasi IN MADRE DE DIOS, PERU}

\author{
Jesús Rojas-Jaimes ${ }^{1, a}$, Edwing Zuñiga- Requena ${ }^{2, b}$, \\ Germán Correa-Nuñez ${ }^{3, c}$
}

Sr. Editor. Los flebotominos o flebótomos son insectos artrópodos del orden Díptera ${ }^{(1)}$. Entre los flebotominos de mayor importancia en salud están los artrópodos del género

\footnotetext{
Escuela de Medicina Humana, Universidad Científica del Sur. Lima, Perú Laboratorio de Entomología, Instituto Nacional de Salud. Lima, Perú.

3 Departamento Académico de Ciencias Básicas, Universidad Nacional Amazónica de Madre de Dios. Madre de Dios, Perú.

a Magister en biología molecular; ${ }^{b}$ biólogo; ${ }^{\mathrm{c}}$ magister en salud ocupacional y medio ambiente

Recibido: 14/12/2017 Aprobado: 17/01/2018 En línea: 28/09/2018
}

Citar como: Rojas-Jaimes J, Zuñiga-Requena E, Correa-Nuñez G. Lutzomyia shannoni un potencial vector de Leishmania chagasi en Madre de Dios, Perú. Rev Peru Med Exp Salud Publica. 2018;35(3): 534-6.doi: 10.17843/ rpmesp.2018.353.3291. 